\title{
Microwave-Assisted Preparation of Polymer Phase-Change Materials
}

\author{
Zhiliang Gao \\ School of Material Science \& Engineering \\ Beifang University of Nationalities \\ Yinchuan, China \\ e-mail: gaozhiliang1993@163.com \\ Enhui Du \\ School of Material Science \& Engineering \\ Beifang University of Nationalities \\ Yinchuan, China \\ e-mail: 515733719@qq.com \\ Liru Zhang \\ School of Material Science \& Engineering \\ Beifang University of Nationalities \\ Yinchuan, China \\ e-mail: zlr2015@yeah.net
}

\author{
Meng Liu \\ School of Material Science \& Engineering \\ Beifang University of Nationalities \\ Yinchuan, China \\ e-mail: 295961108@qq.com \\ Xiang Huang \\ School of Material Science \& Engineering \\ Beifang University of Nationalities \\ Yinchuan, China \\ e-mail: 1063870948@qq.com
}

\begin{abstract}
Phase-change materials have attracted more and more attention in the fields of both academic research and industrial application. To develop a simple, efficient and environment-friendly method for preparing polymer phasechange materials, microwave-assisted technology was employed to synthesis urea-formaldehyde phase-change microcapsules, with the core of dodecyl alcohol, the shell of urea-formaldehyde resin, the emulsifier of Span-80 and Tween-20. A few of key factors, such as emulsifiers, reaction temperature and time, can influence the thermal performance and the encapsulation. Finally, dynamic light scattering (DLS) and differential scanning calorimetry (DSC) were employed to characterize the size distribution, the phase transition temperature and latent heat. The results show that the size of urea-formaldehyde microcapsules is close to $1 \mu \mathrm{m}$. And the urea-formaldehyde phase-change microcapsules have relative high phase-change latent heat storage capacity, $132.3 \mathrm{~J} / \mathrm{g}$ and an encapsulation efficiency of $63.9 \%$, respectively. Microwave-assisted technology has a bright future in the preparation of new-type polymer functional materials.
\end{abstract}

Keywords- microwave-assisted; phase-change; preparation; in situ polymerization; energy storage

\section{INTRODUCTION}

Large amounts of latent heat could be absorbed and released in the process of phase transition of phase-change energy storage materials. This kind of material has been widely used in the fields of energy storage, heat exchange and temperature control, etc. Compared with the methods of sensible heat storage, the storage of latent heat has many advantages: high energy storage density, small temperature difference in heat exchange and less energy loss, etc [1-3]. The research about the encapsulation of phase-change materials was started in the late 1970s. At that time, NASA first proposed the project and used it in the textile industry. And then they applied for a patent successfully. From then, more and more scientists attend to the relative studies [4-8]. Cho et al. synthesized the polymer microcapsules as the core of octadecane and the shell of polyurea through interfacial polymerization. The monomer was toluene diisocyanate (TD1) and dimethyl diethylene triamine (DETA), respectively. And the emulsifier was OP-10. Wang et al. synthesized the polymer microcapsules as the core of octadecane and the shell of the copolymer of formaldehyde and melamine through in situ polymerization. They investigated the influence of $\mathrm{pH}$ value to the stability of the reaction system, the surface morphology and thermal performance of polymer microcapsules during the process of polymerization. Microwave-assisted heating is a technology of spreading electromagnetic wave inside the object. The internal and the external of objects are heated at the same time. So it has advantages of perfect heating rate, uniform heating and easy control, etc. In this paper, urea-formaldehyde phasechange microcapsules were prepared through microwaveassisted in situ polymerization method.

\section{EXPERIMENTAL}

\section{A. Materials}

Urea, triethanolamine and dodecyl alcohol were purchased as analytical grade from Tianjin Guangfu Fine Chemicals Institute, China. Formaldehyde (37.0-40.0 wt \%) 
was purchased as analytical grade from Xuzhou Tianhong Chemicals Co., Ltd., China. Tween-20 was purchased as chemical grade from Sinopharm Group Co., Ltd., China. Span-80 was purchased as chemical grade from Aladdin. They all were used as received.

\section{B. Apparatus}

The equipment employed in this research is a MAS II microwave synthesis system (Shanghai Xinyi Microwave Chemistry Technology Co., Ltd.). During the polymerization, thermostated water was circulated to maintain the temperature constant.

\section{Preparation of urea-formaldehyde/dodecyl alcohol phase-change microcapsules through microwave- assisted in situ polymerization.}

A certain amount of urea and formaldehyde was stirred to dissolve the former. And then, triethanolamine was used to adjust the $\mathrm{pH}$ value to $8.5 \sim 9.0$. At $82{ }^{\circ} \mathrm{C}$, the mixture was stirred for 1 hour by $1500 \mathrm{r} / \mathrm{min}$. And the ureaformaldehyde prepolymer was obtained (diluted with distilled water). A certain amount of surfactant (the mixture of Span-80 and Tween-20), dodecyl alcohol and distilled water was homogenized for $30 \mathrm{~min}$ by $4000 \mathrm{r} / \mathrm{min}$ and poured into the special vessel. The urea-formaldehyde prepolymer was put into the constant pressure funnel and evenly dripped during the reaction process. The microwave reaction parameters (temperature, time, power and stirring speed) were set in advance. The obtained emulsion was cooled, demulsified, filtrated, washed and dried. And the ultimate product was just phase-change polymer microcapsules.

\section{Characterization}

The average microcapsule size (z-average size) and size distribution were measured by photon correlation spectroscopy (Nano ZS90 zetasizer, Malvern Instruments Corp, U.K.) at $25{ }^{\circ} \mathrm{C}$ under a fixed angle of $90^{\circ}$ in disposable polystyrene cuvettes. The measurements were obtained using a He-Ne laser of $633 \mathrm{~nm}$. No multiscattering phenomenon was observed. The thermal properties of urea-formaldehyde/dodecyl alcohol phasechange microcapsules such as latent heat storage capacity and melting point were obtained by differential scanning calorimetry (DSC, TA instruments Q20). DSC measurements were performed at a $10{ }^{\circ} \mathrm{C} / \mathrm{min}$ heating and cooling rate. Furthermore, the samples were heated from $0{ }^{\circ} \mathrm{C}$ to $80{ }^{\circ} \mathrm{C}$ and cooled from $80{ }^{\circ} \mathrm{C}$ to $0{ }^{\circ} \mathrm{C}$ under a nitrogen atmosphere. With DSC measurements, heating and cooling curves were obtained. By analyzing these curves, the thermal properties and heat storage capacity of urea-formaldehyde/dodecyl alcohol phase-change microcapsules were determined.

\section{RESULTS AND DISCUSSION}

\section{A. DLS analysis}

Dynamic light scattering (DLS) is the most important technique for directly detecting cluster populations in solution [9]. It uses photon correlation spectroscopy (PCS) and consists of analyzing the time fluctuations of the light scattered by optically anisotropic particles subjected to Brownian motion in a solvent of a given viscosity. The Monochromatic laser light scattered by a solution at a given angle is analyzed in terms of the temporal field autocorrelation function $\mathrm{C}(\tau)$ sampled over a wide range of times $\tau$. Considering this motion in terms of translational and rotational diffusion, the dimensions of the particles can be determined as a drastic change in the time-intensity correlation function. By contrast with imaging techniques, DLS does not need the drying of the dispersion and the measurements are performed faster.

When the microwave-assisted in situ polymerization was finished, the emulsion was diluted to an appropriate concentration with deionized water. The particle size and distribution of urea-formaldehyde/dodecyl alcohol phasechange microcapsules are presented in Fig. 1. The average size of polymer microcapsules is around $950 \mathrm{~nm}$. And the polydispersity index (PDI) is 0.588. Furthermore, correlation function is a statistical method to study fluctuation phenomenon of PCS. In DLS characterization, the measured baseline and the calculated baseline are highly coincident. That is to say, the optical purification of samples is in good quality. It shows a better fitting and the testing results are believable.

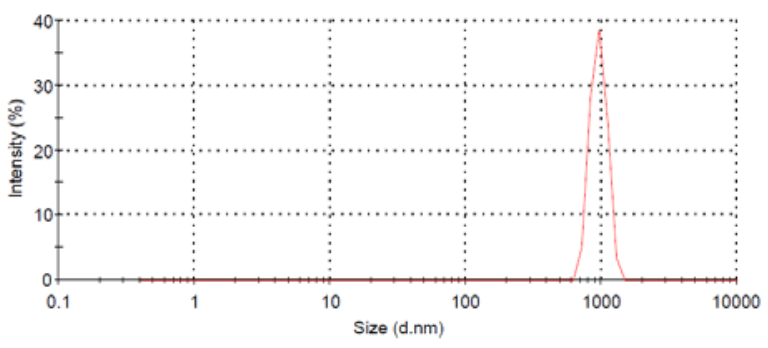

Figure 1 Size and distribution of urea-formaldehyde/dodecyl alcohol phase-change microcapsules prepared through microwave-assisted in situ polymerization

\section{B. DSC analysis}

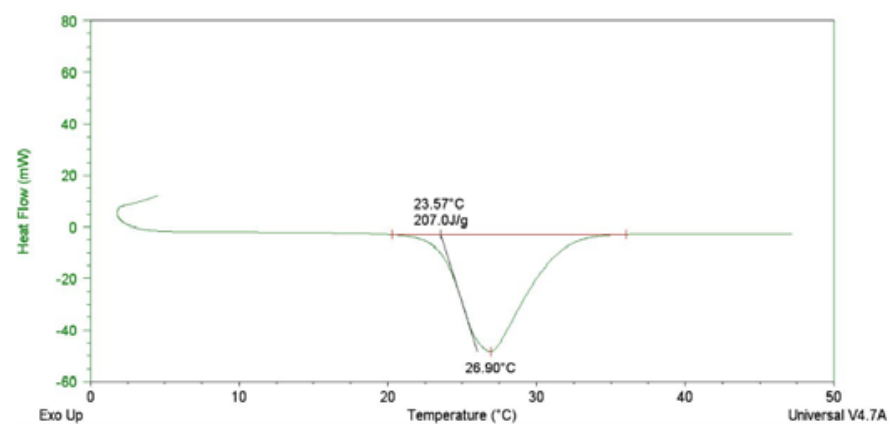

Figure 2 DSC curve of pure dodecyl alcohol

Fig. 2 and Fig. 3 shows the typical DSC thermograms of pure dodecyl alcohol and ureaformaldehyde/dodecyl alcohol phase-change microcapsules prepared by microwave-assisted in situ polymerization. The thermal properties of pure dodecyl alcohol and urea-formaldehyde/dodecyl alcohol phasechange microcapsules, such as the onset transition temperature $\left(T_{\text {onset }}\right)$, the peak temperature $\left(T_{\text {peak }}\right)$ and the latent heat storage capacity $\left(\triangle H_{\text {latent }}\right)$ obtained by the DSC measurements are given. From the DSC curves in Fig. 2, the $T_{\text {onset }}, T_{\text {peak }}$ and $\triangle H_{\text {latent }}$ of pure dodecyl alcohol 
are found to be $23.57{ }^{\circ} \mathrm{C}, 26.90{ }^{\circ} \mathrm{C}$ and $207.0 \mathrm{~J} / \mathrm{g}$, respectively. But to urea-formaldehyde/dodecyl alcohol phase-change microcapsules in Fig. 3, the above specifications are $22.62{ }^{\circ} \mathrm{C}, 26.19{ }^{\circ} \mathrm{C}$ and $132.3 \mathrm{~J} / \mathrm{g}$, respectively. Encapsulated stearic acid shows a phase change over the similar temperature range as that of the bulk. However, the $T_{\text {onset }}$ and $T_{\text {peak }}$ of ureaformaldehyde/dodecyl alcohol phase-change microcapsules are both lower than that of pure core materials. Moreover, as it is understood from the obtained data, to the latent heat storage capacity, $\triangle H_{\text {latent }}$ of ureaformaldehyde/dodecyl alcohol phase-change microcapsules is also decreased owing to the encapsulation of the polymer shell around the core materials compared with that of pure dodecyl alcohol.

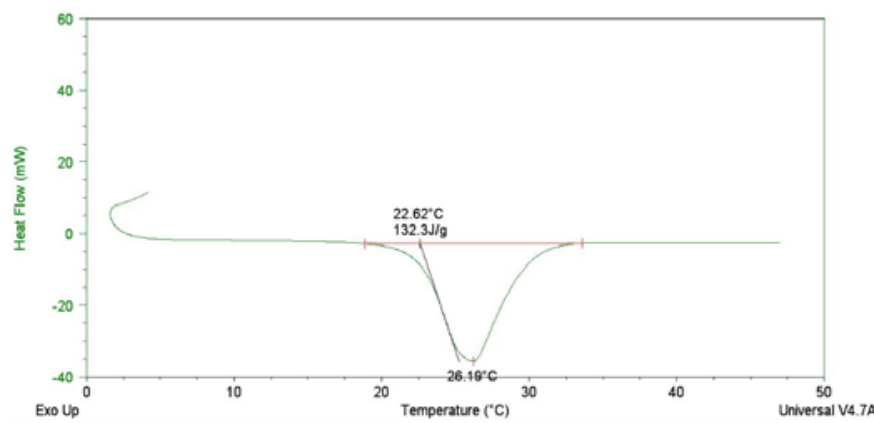

Figure 3 DSC curve of urea-formaldehyde/dodecyl alcohol phase-change microcapsules

It should be noted that, in the process of encapsulation, calculation of encapsulation efficiency is very important. And in our study, the encapsulation efficiency of ureaformaldehyde/dodecyl alcohol phase-change microcapsules was calculated by the way of the enthalpy values of urea-formaldehyde/dodecyl alcohol and dodecyl alcohol, following the equation [10]:

Encapsulation efficiency $\%=\triangle H_{\text {microcap sule }} / \triangle H_{\text {dodecyl alcohol } * 1}$

And then, the encapsulation efficiency of microwaveassisted in situ polymerization reaction could be determined as $63.9 \%$.

C. Inflence of emulsifiers

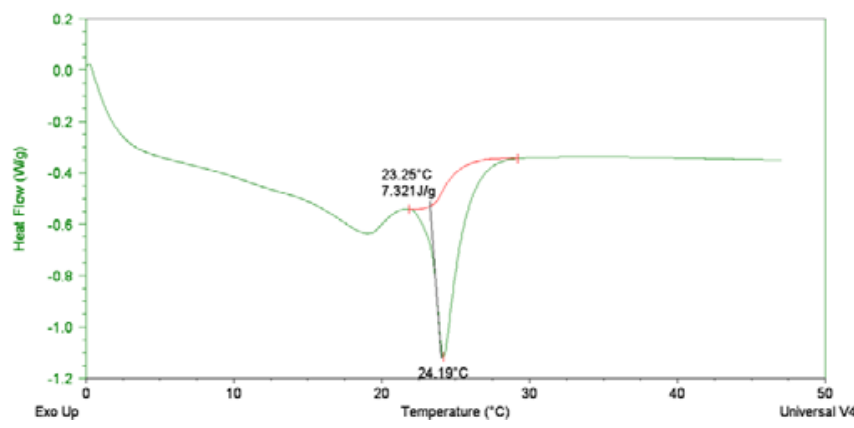

Figure 4 DSC curve of urea-formaldehyde/dodecyl alcohol phase-change microcapsules (SDS as an emulsifier)

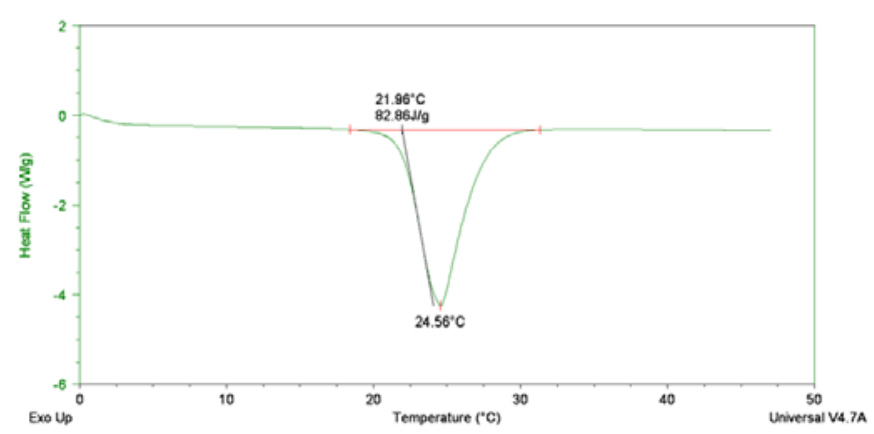

Figure 5 DSC curve of urea-formaldehyde/dodecyl alcohol phase-change microcapsules (Brij-56 as an emulsifier)

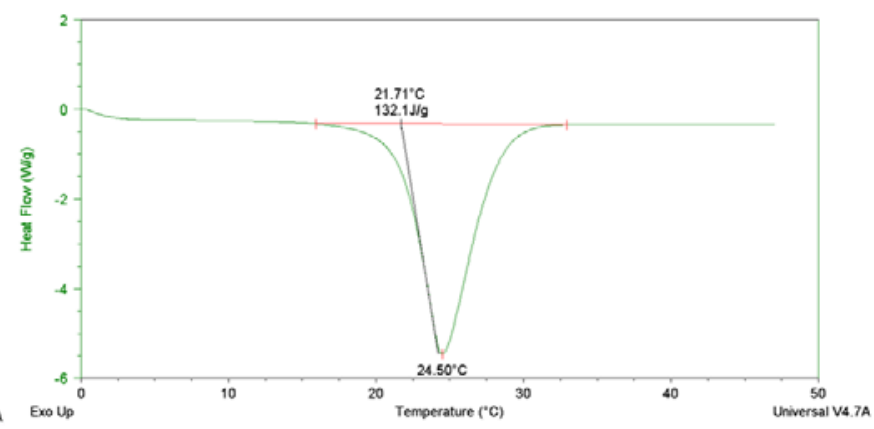

Figure 6 DSC curve of urea-formaldehyde/dodecyl alcohol phase-change microcapsules (OP-10 as an emulsifier)

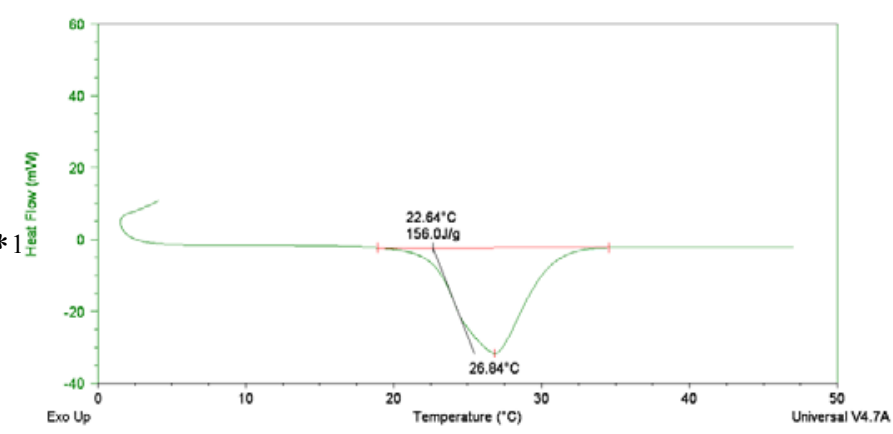

Figure 7 DSC curve of urea-formaldehyde/dodecyl alcohol phase-change microcapsules (composite emulsifier of Span-80 and Tween-20 as an emulsifier)

In the preparation of urea-formaldehyde/dodecyl alcohol phase-change microcapsules, the emulsifier, including the type and the amount, is a very important factor. It can influence both the morphology and the latent heat storage capacity of polymer phase-change microcapsules directly. Therefore, we discussed the effect of different emulsifiers on the latent heat storage capacity of urea-formaldehyde/dodecyl alcohol phase-change microcapsules. We chose four emulsifiers, sodium dodecyl sulfate (SDS, anion surfactant), Brij-56 (nonionic surfactant), polyoxyethylene octylphenol ether (OP10 , non-ionic surfactant) and the mixture of Tween-20 and Span-80 (non-ionic surfactant). From Fig. 4 to Fig. 7, it is obvious that the urea-formaldehyde/dodecyl alcohol phase-change microcapsules made from composite 
emulsifier of Span-80 and Tween-20 has the best latent heat storage capacity $(156.0 \mathrm{~J} / \mathrm{g})$. And that of materials made from SDS is the worst (only $7.3 \mathrm{~J} / \mathrm{g}$ ). Therefore, single emulsifier is not enough to obtain the desirable product. That is, we must adjust the HLB value of emulsifiers. Furthermore, there is a little difference among the $T_{\text {peak }}$ of the urea-formaldehyde/dodecyl alcohol phase-change microcapsules. The $T_{\text {peak }}$ of the ureaformaldehyde/dodecyl alcohol phase-change microcapsules made from composite emulsifier $\left(26.84{ }^{\circ} \mathrm{C}\right)$ is slight higher than that of materials made from the other emulsifiers. But the other three almost have the same $T_{\text {peak }}$.

\section{CONCLUSION}

The urea-formaldehyde/dodecyl alcohol phase-change microcapsules were obtained by applying microwaveassisted in situ polymerization. The size of polymer microcapsules is close to $1 \mu \mathrm{m}$. The ureaformaldehyde/dodecyl alcohol phase-change microcapsules have relative high phase-change latent heat storage capacity, $132.3 \mathrm{~J} / \mathrm{g}$ and an encapsulation efficiency of $63.9 \%$, respectively. The type of the emulsifiers has very important influence on the latent heat energy capacity of the urea-formaldehyde/dodecyl alcohol phase-change microcapsules. And the composite emulsifier is the best choice. Microwave-assisted technology is simple, efficient and environment-friendly. It provides a new choice to prepare phase-change energy storage materials.

\section{ACKNOWLEDGMENT}

This work was financially supported by National Undergraduates Innovating Experimentation Project (Grant No. 201411407027).

\section{REFERENCES}

[1] J. Choi, J. Lee, and J. Kim, "Preparation of microcapsules containing phase change materials as heat transfer media by in-situ polymerization,” J. Ind. Eng. Chem., Vol. 7, 2001, pp. 358-362.

[2] E. Fallahi, M. Barmar, and M. H. Kish, "Preparation of phasechange material microcapsules with paraffin or camel fat cores: application to fabrics," Iran. Polym. J., Vol. 19, 2010, pp. 277-286.

[3] Y. Zhao, W. Zhang, L. P. Liao, W. J. Li, and Y. Xin, "Preparation process of microcapsule containing phase change material for thermal-energy storage," Adv. Sci. Lett., Vol. 4, 2011, pp. 933-937.

[4] D. Zhou, C. Y. Zhao, and Y. Tian, "Review on thermal energy storage with phase change materials (PCMs) in building applications," Appl. Energ., Vol. 92, 2012, pp. 593-605.

[5] E. Oró, A. de Gracia, A. Castell, M.M. Farid, and L.F. Cabeza, "Review on phase change materials (PCMs) for cold thermal energy storage applications,” Appl. Energ., Vol. 99, 2012, pp. 513533.

[6] L. W. Fan, and J. M. Khodadadi, "Thermal conductivity enhancement of phase change materials for thermal energy storage: A review,” Renew. Sust. Energ. Rev., vol. 15, 2011, pp. 24-46.

[7] M. M. Kenisarina, and K. M. Kenisarinab, "Form-stable phase change materials for thermal energy storage," Renew. Sust. Energ. Rev., vol. 16, 2012, pp. 1999-2040.

[8] S. Peng, A. Fuchs, and R. A. Wirtz, "Polymeric phase change composites for thermal energy storage," J. Appl. Polym. Sci., vol. 93, 2004, pp. 1240-1251.

[9] G. K. Min, M. A. Bevan, D. C. Prieve, and G. D. Patterson, "Light scattering characterization of polystyrene latex with and without adsorbed polymer," Coll. Surf. A: Physiochem. Eng. Aspects, vol. 202, 2002, pp. 9-21.

[10] C. Alkan, A. Sari, A. Karaipekli, and O. Uzun, "Preparation, characterization and thermal properties of microencapsulated phase change material for thermal energy storage," Sol. Energy Mater. Sol. Cells, vol. 93, 2009, pp. 143-147. 